\title{
BAK1 Is Not a Target of the Pseudomonas syringae Effector AvrPto
}

\author{
Tingting Xiang, ${ }^{1,2} \mathrm{Na}$ Zong, ${ }^{2}$ Jie Zhang, ${ }^{2}$ Jinfeng Chen, ${ }^{3}$ Mingsheng Chen, ${ }^{3}$ and Jian-Min Zhou ${ }^{2}$ \\ ${ }^{1}$ State Key Laboratory of Plant Physiology and Biochemistry (SKLPPB), College of Biological Sciences, China Agricultural \\ University; ${ }^{2}$ National Institute of Biological Sciences, Beijing; ${ }^{3}$ State Key Laboratory of Plant Genomics, Institute of Genetics \\ and Developmental Biology, Chinese Academy of Sciences, Beijing 100101, China
}

Submitted 28 April 2010. Accepted 24 September 2010.

\begin{abstract}
Plant cell surface-localized receptor kinases such as FLS2, EFR, and CERK1 play a crucial role in detecting invading pathogenic bacteria. Upon stimulation by bacterium-derived ligands, FLS2 and EFR interact with BAK1, a receptorlike kinase, to activate immune responses. A number of Pseudomonas syringae effector proteins are known to block immune responses mediated by these receptors. Previous reports suggested that both FLS2 and BAK1 could be targeted by the $P$. syringae effector AvrPto to inhibit plant defenses. Here, we provide new evidence further supporting that FLS2 but not BAK1 is targeted by AvrPto in plants. The AvrPto-FLS2 interaction prevented the phosphorylation of BIK1, a downstream component of the FLS2 pathway.
\end{abstract}

The ability of plants to recognize invading pathogens and activate immune responses is critical for plant survival. As the first layer of induced immunity, plants use cell surface-localized pattern-recognition receptors (PRR), typically receptor kinases or receptor-like proteins, to detect conserved microbial molecular signatures termed pathogen- or microbe-associated molecular patterns (PAMPs or MAMPs), thereby activating PAMP-triggered immunity (PTI) (Chisholm et al. 2006; Zipfel 2008, 2009). For example, the Arabidopsis receptor kinases FLS2 and EFR are receptors of bacterial flagellar peptide flg22 and elongation factor-Tu peptide elf18, respectively (Chinchilla et al. 2006; Zipfel et al. 2006). Upon exposure to ligands, FLS2 and EFR rapidly associate with another receptor-like kinase, BAK1, which is also known to be required for BRI-mediated brassinosteroid (BR) signaling (Chinchilla et al. 2007; Heese et al. 2007, Schulze et al. 2010). Arabidopsis mutants defective in PRR or PTI signaling components are compromised in resistance to pathogens (Gimenez-Ibanez et al. 2009; Miya et al. 2007; Wan et al. 2008; Zipfel et al. 2004, 2006). Conversely, heterologous expression of Arabidopsis EFR in solanaceous plants resulted in increased resistance to several bacterial pathogens (Lacombe et al. 2010). More importantly, recent studies showed that PRR and PTI signaling components are frequently targeted by pathogen virulence factors, indicating that PTI is critically important for plant disease resistance (Fu et al. 2007; Gimenez-Ibanez et al. 2009; Gohre et al. 2008; Xiang et al. 2008; Zhang et al. 2007, 2010; Wang et al. 2010).

The Pseudomonas syringae type III secretion system delivers approximately 30 effector proteins into the host cell. It is

Corresponding author: J.-M. Zhou; E-mail: zhoujianmin@nibs.ac.cn

* The $e$-Xtra logo stands for "electronic extra" and indicates that five supplementary figures and one supplementary table are published online. well-established that many of these effector proteins target important host components to defeat plant immunity (Block et al. 2008; Boller and He 2009; Gohre and Robatzek 2008; Zhang and Zhou 2010; Zhou and Chai 2008). Among these effectors, AvrPto and AvrPtoB have been investigated in greater details. In tomato plants, they both interact with the protein kinase Pto and trigger disease resistance specified by the corresponding resistance protein Prf (Abramovitch et al. 2003; Tang et al. 1996). Prf constitutively interacts with Pto and is inactive in the absence of AvrPto or AvrPtoB (Mucyn et al. 2006). The interaction of Pto with AvrPto or AvrPtoB presumably alters the conformation of the Pto-Prf complex, switching Prf into an active state (Wu et al. 2004; Xing et al. 2007). In susceptible tomato and Arabidopsis plants, AvrPto and AvrPtoB are important virulence determinants (Abramovitch et al. 2003; Shan et al. 2000). We showed that AvrPto can interact with both FLS2 and EFR in vitro and in vivo and that this interaction is correlated with the virulence function of AvrPto (Xiang et al. 2008). The functional significance of FLS2-AvrPto interaction was further supported by the restoration of virulence to an avrPto-deficient strain of $P$. syringae by an fls 2 mutation in Arabidopsis plants. Recent findings from other groups indicate that AvrPtoB also targets PRR (Gimenez-Ibanez et al. 2009; Gohre et al. 2008). The AvrPtoB protein consists of an $\mathrm{N}$-terminal domain and a C-terminal E3 ligase (Abramovitch et al. 2006; Janjusevic et al. 2006), and the E3 ligase activity was shown to mark FLS2 (Gohre et al. 2008) or the putative chitin receptor CERK1 for degradation (Gimenez-Ibanez et al. 2009). Together these studies indicate that AvrPto and AvrPtoB both target PRR to enhance bacterial virulence.

Contrary to the studies described above, Shan and associates (2008) reported that AvrPto interacted with BAK1 more strongly than with FLS2 and suggested that BAK1 but not FLS2 is a physiological target for AvrPto. In this study, we performed additional experiments to thoroughly test the interactions between AvrPto and FLS2, AvrPto and BAK1, and FLS2 and BAK1. The results show that FLS2 but not BAK1 is targeted by AvrPto.

\section{RESULTS}

Interaction of AvrPto with FLS2 and BAK1 in protoplasts.

The ligand-induced formation of the FLS2-BAK1 receptor complex enables the activation of downstream signaling (Wang et al. 2008). An interaction of AvrPto with either FLS2 or BAK1 can explain its inhibition of flg22-induced signaling events and defense responses. To determine if AvrPto preferentially interacts with FLS2 or BAK1, we coexpressed FLAG- 
tagged FLS2 and BAK1 along with a hemagglutinin (HA)tagged AvrPto in Arabidopsis protoplasts and performed coimmunoprecipitation (co-IP) assays. Figure 1A shows that both FLS2-FLAG and BAK1-FLAG accumulated to a similar level in protoplasts. Proteins isolated by anti-HA IP contained only FLS2-FLAG but not BAK1-FLAG, supporting the idea that only FLS2 is targeted by AvrPto. It should be noted that, in our experiments, the immunoprecipitated beads were stringently washed to prevent nonspecific interactions. As a result, not even a trace amount of FLS2 signal was seen when IP was performed in the absence of AvrPto. To further verify this result, we constructed HA-tagged FLS2 and BAK1 and coexpressed them with FLAG-tagged AvrPto. Anti-FLAG IP showed that only FLS2 was detected in the AvrPto immune complex (Fig. 1B). To test if a BAK1-AvrPto interaction could be detected when BAK1 was first immunoprecipitated, we performed anti-HA IP on the protein extract shown in Figure 1B. Again, AvrPto-FLAG was found to associate with FLS2-HA but not BAK1-HA (Supplementary Fig. 1). Thus while the FLS2-AvrPto interaction was reliably detected in co-IP assays, a BAK1-AvrPto interaction was not detected in these experiments.

In some anti-HA co-IP experiments, however, a weak BAK1FLAG signal was detected under long exposures when BAK1FLAG, FLS2-FLAG, and AvrPto-HA were coexpressed in the same protoplasts but not in the absence of FLS2-FLAG overexpression (Fig. 1C). This result suggests that overexpression of FLS2 and BAK1 in the same cell might have caused a low level of ligand-independent FLS2-BAK1 interaction that led to an indirect interaction between BAK1 and AvrPto. To further
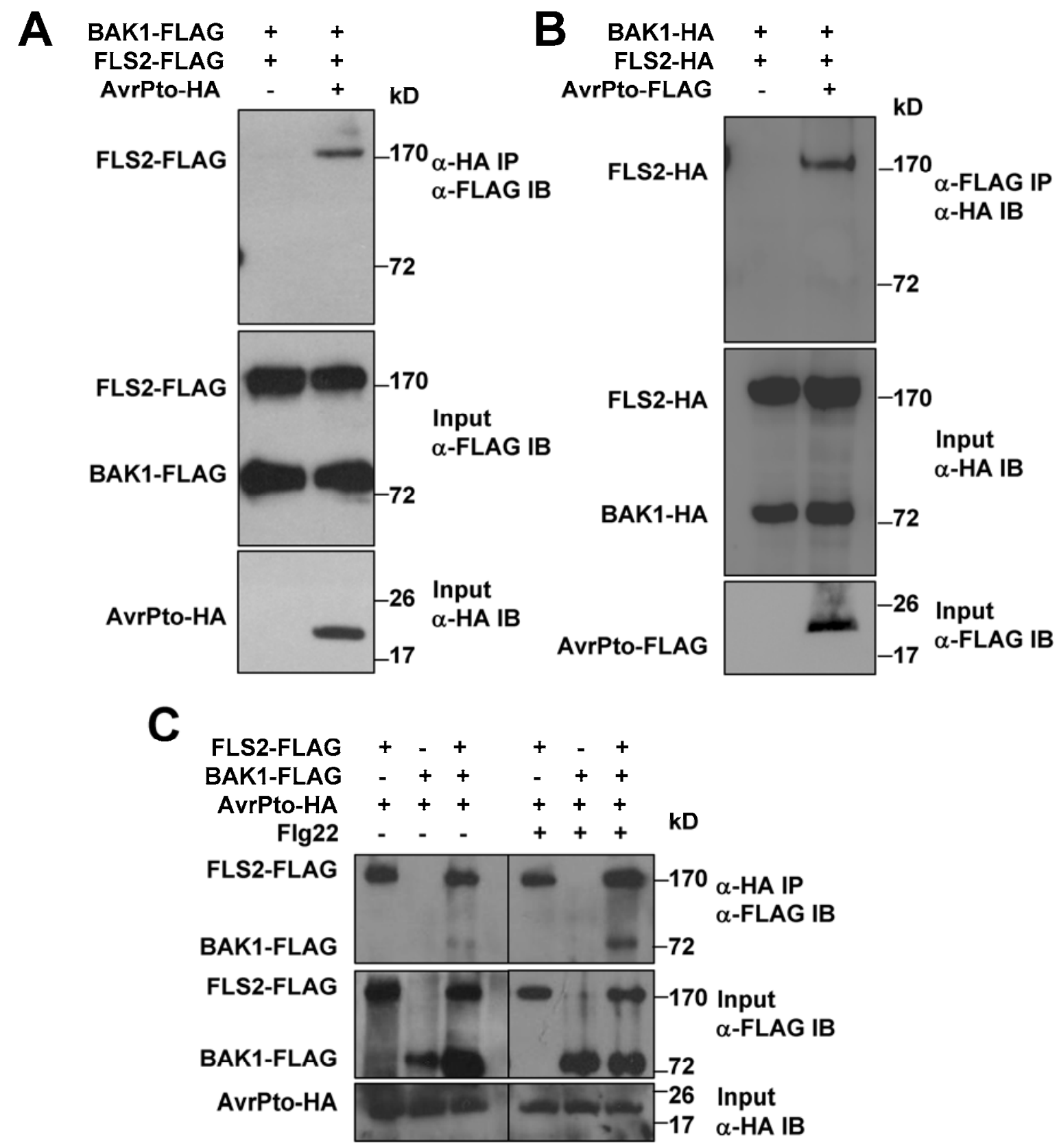

Fig. 1. Coimmunoprecipitation (co-IP) assays for AvrPto-FLS2 and AvrPto-BAK1 interactions in protoplasts. A, FLAG-tagged FLS2 but not BAK1 interacts with hemagglutinin (HA)-tagged AvrPto. The experiment was repeated three times with similar results. B, HA-tagged FLS2 but not BAK1 interacts with FLAG-tagged AvrPto. The experiment was repeated six times with similar results. C, Flg22 treatment recruits BAK1 into the AvrPto-FLS2 complex. Protoplasts were treated with $1 \mu \mathrm{M}$ flg 22 for 5 min before co-IP was performed. The experiment was repeated three times with similar results. IP = immunoprecipitation, IB = immunoblot. 
test this possibility, we treated these protoplasts with flg22 prior to co-IP. Indeed, the flg22 treatment significantly enhanced the BAK1-FLAG signal in the immune complex (Fig. 1C).

In previous studies (Shan et al. 2008; Xiang et al. 2008), the FLS2-AvrPto and BAK1-AvrPto interactions in the plant cell were examined only with co-IP assays. Because co-IP assays involve multiple steps of sample handling, the procedures may be prone to lab-to-lab variations. This may, in part, explain the inconsistency of results between the two published studies. Therefore, we performed bimolecular fluorescence complementation (BiFC) assays (Walter et al. 2004), which involve relatively few steps of sample handling. Expression of the BAK1-cYFP (cytosolic yellow fluorescent protein) fusion protein in bakl protoplasts stimulated FRK1-LUC reporter expression in the presence of flg22 (Supplementary Fig. 2), indicating that our fusion construct was functional. YFP fluorescence was detected only when AvrPto-nYFP (the N-terminal part of YFP) was coexpressed with FLS2-cYFP but not with BAK1-cYFP (Fig. 2), although the protein was expressed at similar levels across different samples. These results further demonstrated that AvrPto is capable of interacting with FLS2 but not BAK1 in plant cells.

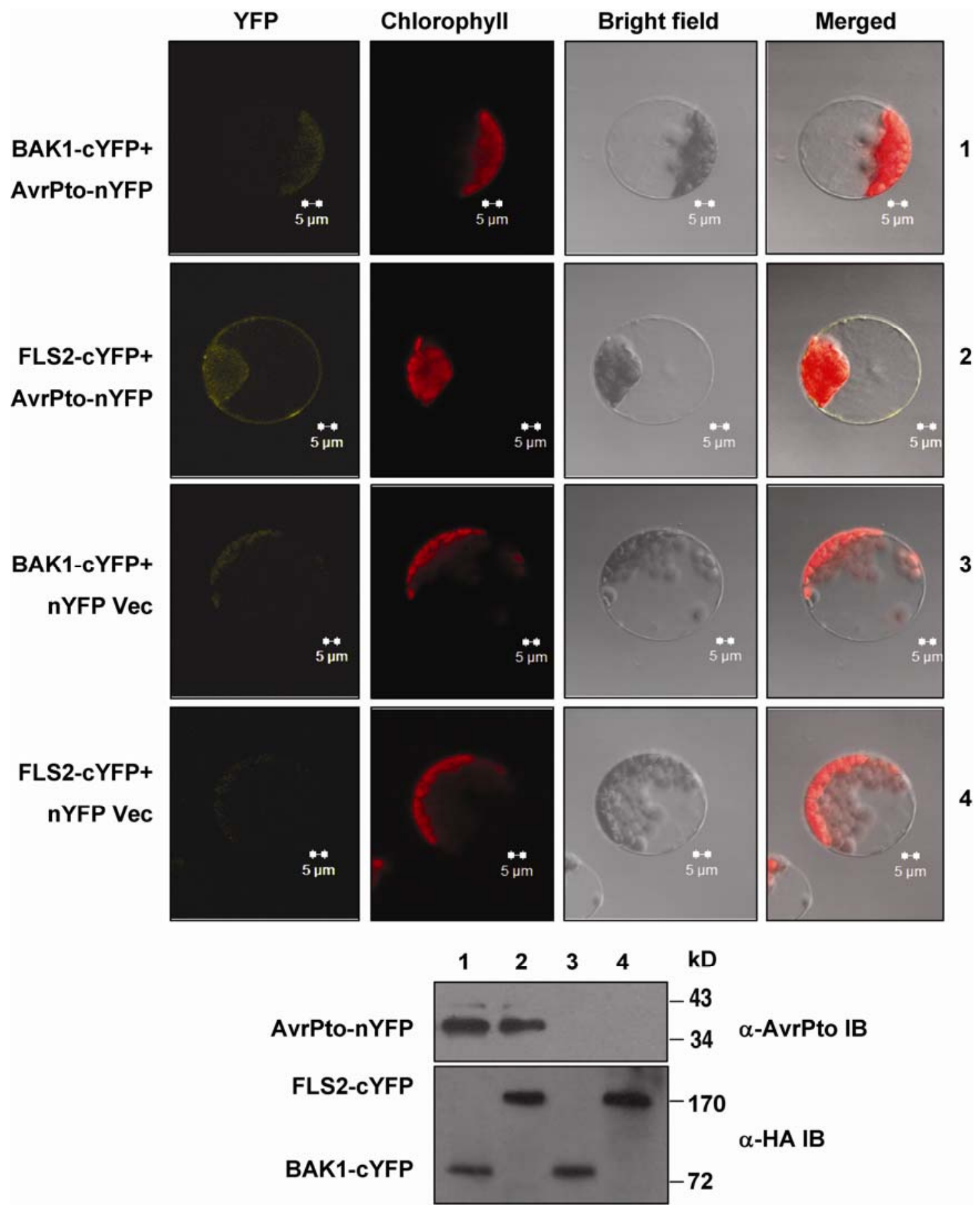

Fig. 2. Bimolecular fluorescence complementation assay for FLS2-AvrPto and BAK1-AvrPto interactions. FLS2-cYFP or BAK1-cYFP were cotransfected with AvrPto-nYFP or the SPYNE vector (nYFP) into protoplasts, and the protoplasts were visualized under a confocal microscope. The images shown are representative of multiple protoplasts (FLS2-cYFP+AvrPto-nYFP, 53; BAK1-cYFP+AvrPto-nYFP, 61; FLS2-cYFP+nYFP, 45; BAK1-cYFP+nYFP, 50). Immunoblots (IB) show protein levels as detected with anti-AvrPto (for AvrPto-nYFP) and anti-HA (hemagglutinin) (for FLS2-cYFP and BAK1-cYFP) antibodies. The experiment was repeated twice with similar results. 
AvrPto interacts with endogenous FLS2 in plants.

Because protoplast assays may or may not reflect physiological conditions in plants, we tested if AvrPto interacts with endogenous FLS2 in transgenic seedlings carrying an estrogeninducible AvrPto-FLAG transgene. Anti-FLAG co-IP experiments specifically detected FLS2 protein in the AvrPto-transgenic seedlings but not nontransgenic seedlings (Fig. 3). AntiBAK1 immunoblot failed to detect BAK1 in the same immune complex (Fig. 3). These results further support that, in plants, AvrPto interacts with FLS2 but not BAK1.

\section{AvrPto can interact with BSK3 and CDG1.}

Transgenic plants overexpressing AvrPto were shown to display BR signaling defects resembling bril and bakl mutants (Shan et al. 2008). This led to the proposal that BAK1 is targeted by AvrPto, because BAK1 is required for both BR signaling and PAMP signaling. We examined morphological phenotypes of seedlings expressing the AvrPto transgene under the estrogen-inducible promoter. The estradiol treatment resulted in severely retarded growth of the AvrPto transgenic seedlings both in the darkness and under light (Supplementary Figs. S3 and S4). Unlike the bakl mutant, which displayed open cotyledons in the dark, the AvrPto-expressing seedlings did not show open cotyledons in the dark but were severely reduced in hypocotyl elongation, a phenotype that is also shared by the bril5 mutant. In addition, root growth was severely arrested in the AvrPto seedlings. The AvrPto mature plants showed epinastic leaves and delayed flowering, which resembled bril mutant phenotypes. Thus, it appears that transgenic expression of AvrPto leads to a pleiotropic phenotype indicative of both BR signaling defects and defects unrelated to BR signaling. Contrary to our observation, Shan and associates (2008) reported an open cotyledon phenotype in dexamethasone-inducible AvrPto transgenic seedlings, a difference possibly explained by the different expression systems used.

It was recently shown that several receptor-like cytoplasmic kinases (RLCK) called BR-signaling kinases (BSK) are substrates of BRI1 and mediate BR signaling (Tang et al. 2008). In addition, another RLCK, CDG1, was also proposed to function in BR signaling (Muto et al. 2004). The inability of AvrPto to interact with BAK1 prompted us to test if AvrPto can interact with BSK or CDG1. Co-IP assays showed that AvrPto can interact strongly with both BSK3 and CDG1 in protoplasts (Fig. 4). A weak interaction with BSK1 was also

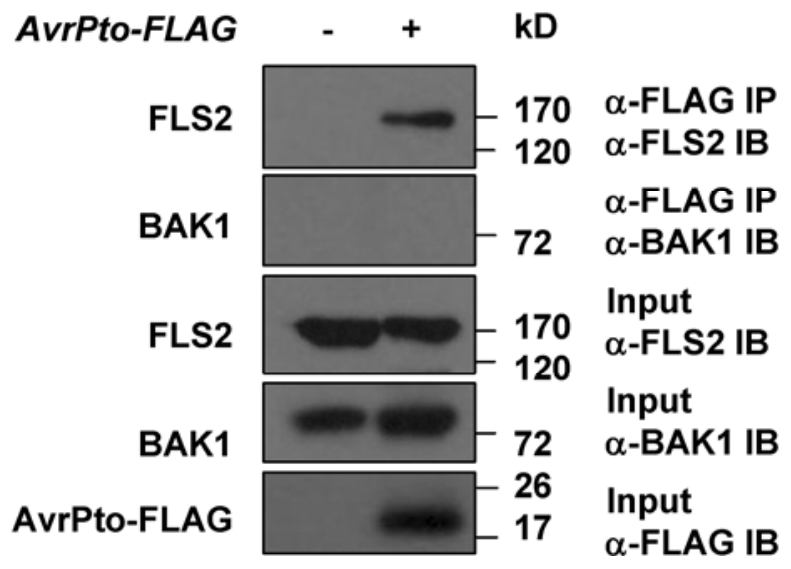

Fig. 3. AvrPto interacts with FLS2 in plants. Eight-day-old seedlings with (+) or without ( - ) the AvrPto-FLAG transgene were induced with $10 \mu \mathrm{M}$ estradiol for 2 days, and membrane protein was extracted for anti-FLAG immunoprecipitation (IP). The presence of FLS2 or BAK1 in the immune complex was determined using anti-FLS2 (eight experiments) or antiBAK1 (two experiments) antibodies. Similar results were obtained in these experiments. $\mathrm{IB}=$ immunoblot. detected. In contrast, BIK1, an RLCK acting downstream of FLS2, EFR, and CERK1 (Lu et al. 2010; Zhang et al. 2010), did not interact with AvrPto (Fig. 4). These results indicate that AvrPto can interact with protein kinases downstream of the BRI1-BAK1 receptor complex, providing an explanation for the observed BR phenotype in AvrPto plants. Pto and Fen belong to the receptor-like kinase (RLK) superfamily and share greatest homology with the CrRPK1L-1 subfamily kinases when aligned with Arabidopsis proteins (Supplementary Fig. 5). AvrPto is capable of interacting with CrRPK1L-1 member At2g23200 (Xiang et al. 2008), RLCK VII member CDG1, RLCK XII members BSK1 and BSK3, and leucine-rich repeat XII members FLS2 and EFR (Xiang et al. 2008). Thus, AvrPto does not appear to target a particular RLK subfamily. It is possible that its specificity is determined by the tertiary structure of the kinases.

\section{AvrPto prevents the phosphorylation of BIK1 but not the ligand-induced FLS2-BAK1 association.}

We previously proposed that AvrPto blocks PAMP signaling by inhibiting the kinase activity of PRR, including FLS2 and EFR (Xiang et al. 2008). We recently showed that BIK1 and its close homolog PBL1 are required for PTI signaling downstream of FLS2, EFR, and CERK1. Flg22 treatment leads to FLS2-dependent phosphorylation of BIK1 in protoplasts and in plants, suggesting that BIK1 is a substrate for FLS2. We also showed that transient expression of AvrPto in protoplasts prevents the flg22-induced phosphorylation on BIK1 (Zhang et al. 2010). To further determine if AvrPto blocks the ligandinduced phosphorylation of BIK1 in plants, we crossed an AvrPto-FLAG transgenic line with a BIK1-HA transgenic line. Figure 5 shows that, while the BIKI-HA plants lacking AvrPto showed a typical flg22-induced BIK1 phosphorylation, the expression of AvrPto abolished this phosphorylation. These results are consistent with a role of AvrPto as a kinase inhibitor.

It was reported that AvrPto impedes the flg22-induced FLS2BAK1 association (Shan et al. 2008). We independently verified

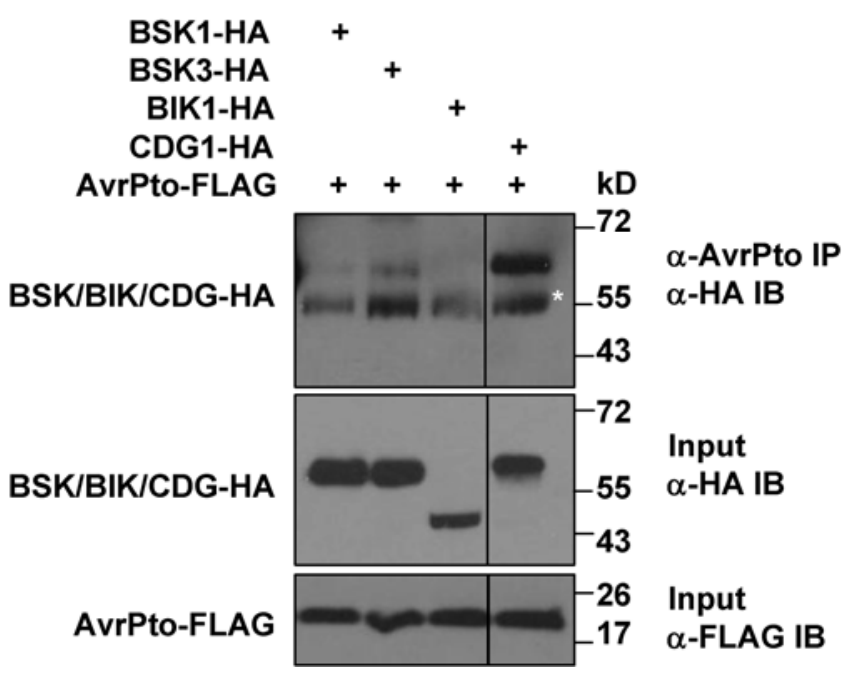

Fig. 4. AvrPto can interact with BSK1, BSK3, and CDG1 but not with BIK1 in protoplasts. Hemagglutinin (HA)-tagged BSK1, BSK3, BIK1, and CDG1 were coexpressed with AvrPto-FLAG in Col-0 protoplasts, and total protein was immunoprecipitated with anti-AvrPto antibodies. The presence of AvrPto-FLAG and HA-tagged proteins in the immune complex was determined by immunoblot (IB), using appropriate antibodies. Asterisk indicates heavy chain of rabbit immunoglobulin $\mathrm{G}(\mathrm{IgG})$. Note that in the coimmunoprecipitation (co-IP) experiment, rabbit anti-AvrPto antibodies were used instead of mouse anti-FLAG antibody to avoid high background signal from mouse IgG heavy chain, which obstructs the observation of BSK, BIK1, and CDG1. The results shown are representative of three experiments. 


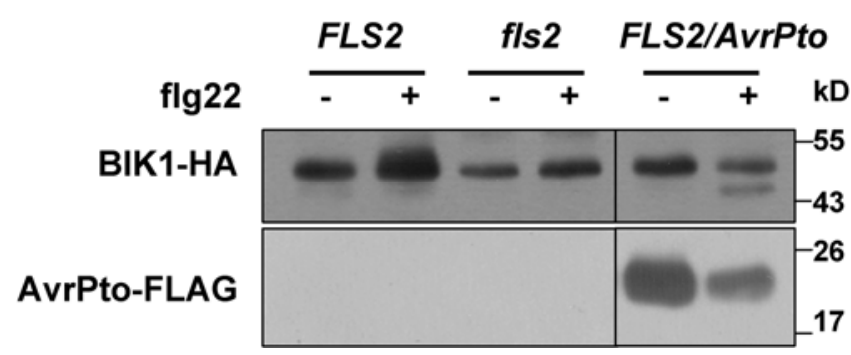

Fig. 5. AvrPto prevents flg22-induced BIK1 phosphorylation in plants. BIK1::BIK1-HA transgenic plants (Zhang et al. 2010) of the indicated genetic background were sprayed with $10 \mu \mathrm{M}$ flg 22 for $10 \mathrm{~min}$, and mobility of BIK1-HA (hemagglutinin) in sodium dodecyl sulfate-polyacrylamide gel electrophoresis was examined with anti-HA immunoblot. The slower migration of BIK1-HA following flg22 treatment is indicative of phosphorylation (Zhang et al. 2010). The experiment was repeated three times with similar results. this result by using co-IP assays in protopolasts. Figure 6A shows that flg22 induced a strong interaction between FLS2FLAG and BAK1-HA either in the presence or absence of the nYFP-tagged AvrPto (Xiang et al. 2008). Likewise, FLS2-HA and BAK1-FLAG also showed flg22-induced association, regardless of the presence or absence of AvrPto-nYFP (Fig. 6B). To unequivocally determine if the PTI-inhibitory activity of AvrPto is correlated with the proposed interference of FLS2BAK1 interaction, we cotransfected the same protoplasts with FLS2-FLAG, BAK1-HA, AvrPto-nYFP, and the FRK1-LUC reporter plasmids. The same protoplasts were assayed for flg22-induced reporter gene expression and FLS2-BAK1 interaction. The expression of AvrPto did not alter viability of the protoplasts but did reduce the FRK1-LUC expression to the background level, indicating that AvrPto completely abolished FLS2-mediated signaling (Fig. 6C). The interaction between FLS2-FLAG and BAK1-HA, however, was normal in the pres-
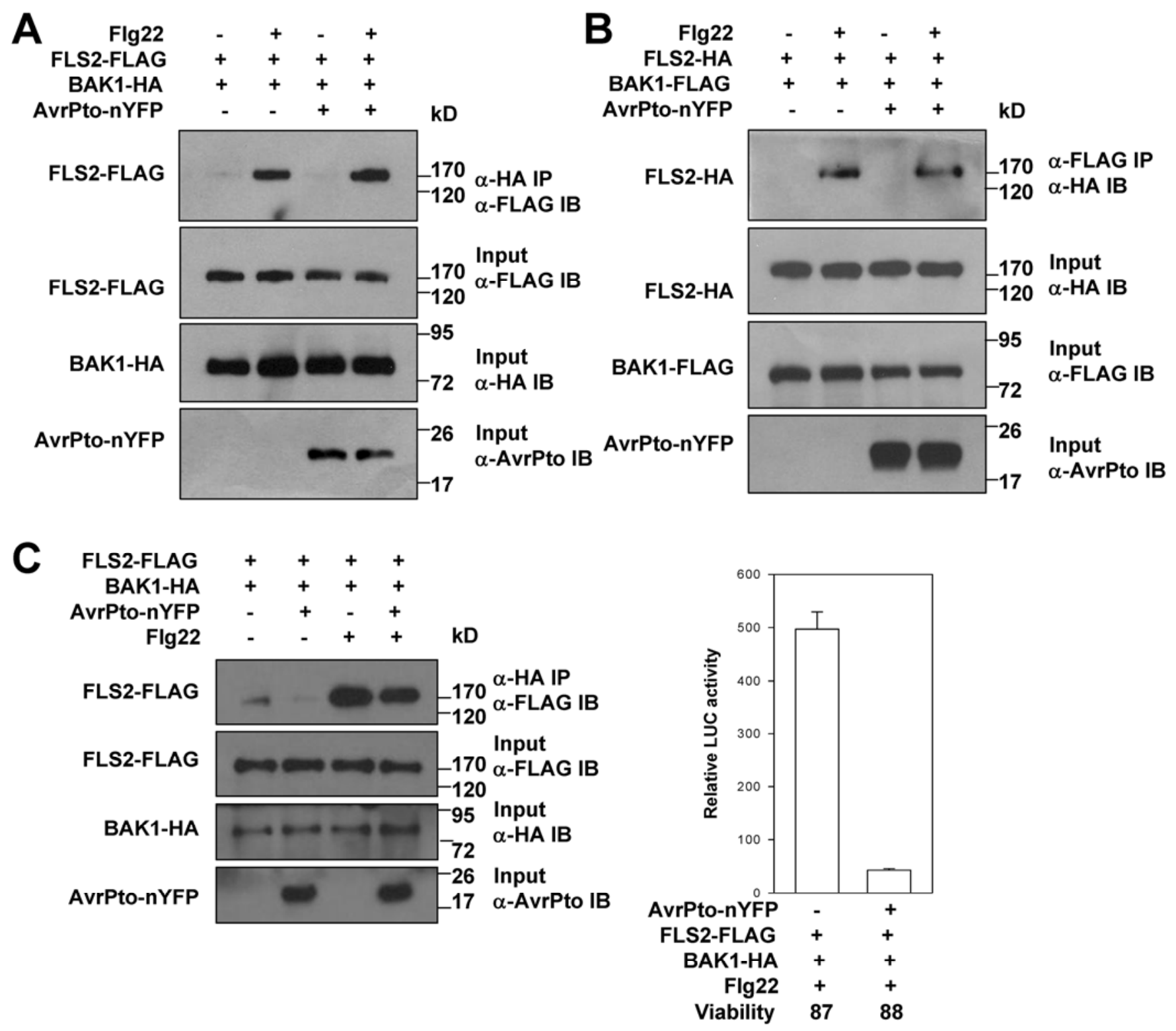

Fig. 6. AvrPto does not interfere with the flg22-induced FLS2-BAK1 interaction. A, Flg22 induces an interaction between FLS2-FLAG and BAK1-HA (hemagglutinin) in the presence of AvrPto-nYFP. B, Flg22 induces an interaction between FLS2-HA and BAK1-FLAG in the presence of AvrPto-nYFP. C, AvrPto-nYFP completely blocks flg22-induced FRK1 expression in the presence of FLS2-BAK1 interaction. Col-0 protoplasts were transfected with the indicated constructs (A to C) and the FRK1-LUC reporter construct (C), were induced with $1 \mu \mathrm{M}$ flg22 for 10 min before protein was extracted for coimmunoprecipitation. A fraction of the protoplasts in C were examined for FRK1-LUC expression and protoplast viability (\%) at $3 \mathrm{~h}$. Error bars indicate standard deviation. The experiments were repeated three times with similar results. 
ence of AvrPto. Thus, our experiments yielded no evidence for an effect of AvrPto on FLS2-BAK1 interaction.

\section{DISCUSSION}

In this study, we show that both the transiently expressed FLS2 and the endogenous FLS2 interact strongly with AvrPto. Our co-IP and BiFC assays on protoplasts always detected a strong AvrPto-FLS2 interaction but not an AvrPto-BAK1 interaction. We occasionally detected a weak BAK1 signal in the AvrPto immune complex only when both BAK1 and FLS2 were overexpressed in the same protoplasts. Furthermore, treatment of these protoplasts with flg22, which induces a strong FLS2-BAK1 interaction, enhanced the BAK1 protein abundance in the AvrPto immune complex. In contrast, the AvrPto-FLS2 interaction is independent of flg22 treatment (Xiang et al. 2008). These results are consistent with our previous findings that AvrPto directly interacts with FLS2 and indicate that the observed AvrPto-BAK1 co-IP occurred indirectly through a FLS2BAK1 interaction.

Our co-IP assay results are in conflict with those of Shan and associates (2008), who reported a preferential interaction of AvrPto with BAK1. Although protoplast-based co-IP assays were performed in both studies, subtle differences in experimental procedures exist. Co-IP assays involve multiple steps of sample handling that could potentially introduce variations from different labs. For example, our co-IP assays involve eight washes of beads containing the immune complex (Xiang et al. 2008; this study), whereas five washes were done by Shan and associates (2008). In addition, our washing buffer contained higher concentrations of Triton X-100 than that of Shan and associates (2008). Different stringencies in the co-IP assays might have caused opposite results. To minimize potential artifacts from sample manipulation during the experiments, we performed BiFC assays, and our results clearly supported an interaction of AvrPto with FLS2 but not BAK1. Thus, our conclusion is supported by protoplast- and plant-based co-IP assays from different orientations and BiFC assays.

It was reported that the AvrPto ${ }^{\mathrm{S} 46 \mathrm{P}}$ mutant interacted with FLS2 but not BAK1 and that this mutant was unable to interfere with the flg22-induced FLS2-BAK1 interaction (Shan et al. 2008). The differential interaction was thought to support the biological significance of AvrPto-BAK1 interaction (Shan et al. 2008). However, this mutant is highly unstable and does not accumulate in $P$. syringae (Shan et al. 2000), a fact that complicates the interpretation of results.

Our results showed that accumulation of a high level of AvrPto in transgenic plants can lead to pleiotropic developmental phenotypes that may be explained, in part, by defects in the BR pathway. Because BAK1 plays a dual role in BR and PAMP signaling, it becomes a logical candidate of AvrPto target. However, our results clearly showed that AvrPto does not interact with BAK1. Instead, AvrPto can interact with other protein kinases required for plant development. For example, AvrPto can interact with the protein kinases BSK3 and CDG1, two RLCK that function in BR signaling (Muto et al. 2004; Tang et al. 2008), suggesting that overexpression of AvrPto in plants can potentially block BR signaling downstream of BRI1 and BAK1. This provides an alternative explanation for the observed BR signaling defects and suggests that developmental phenotypes of AvrPto-transgenic plants do not necessarily provide an accurate prediction of its physiological targets.

Shan and associates (2008) suggested that AvrPto physically impedes the flg22-induced FLS2-BAK1 association. However, our analyses failed to detect such an effect, although AvrPto completely blocked PTI signaling in the same protoplasts. We previously showed that AvrPto can act as a kinase inhibitor to block FLS2 and EFR kinase activity, at least in vitro (Xiang et al. 2008). This is consistent with the finding that AvrPto binds Pto as a pseudosubstrate (Xing et al. 2007). AvrPto displayed significant kinase inhibitory activity when AvrPto and Pto are mixed at a ratio of approximately 1:1, and the kinase inhibition activity requires AvrPto tyrosine 89, which is essential for AvrPto-Pto and AvrPto-FLS2 interaction (Xiang et al. 2008; Xing et al. 2007). These results indicate that AvrPto inhibits protein kinases through a direct protein-protein interaction. We recently showed that the BIK1 directly interacts with FLS2, EFR, and CERK1 to mediate PTI. BIK1 is phosphorylated in vivo upon flg22 stimulation in a FLS2-dependent manner, suggesting that BIK1 is a substrate of FLS2. This phosphorylation is blocked by AvrPto in both protoplasts and plants. Because AvrPto does not interact with BIK1 in vivo, it is likely that AvrPto inhibits the kinase activity of FLS2 to block BIK1 phosphorylation.

Taken together, our extensive analyses on protein-protein interactions among AvrPto, FLS2, and BAK1 in previous reports and this study clearly indicate that FLS2 but not BAK1 is targeted by AvrPto. The FLS2-AvrPto interaction leads to a blockage of phosphorylation events downstream of FLS2.

\section{MATERIALS AND METHODS}

Plants.

Plants used in this study include the bakl-4 (Chinchilla et al. 2007), fls2 (Xiang et al. 2008), and bril-5 (Noguchi et al. 1999) mutants, BIK1-HA (Zhang et al. 2010) and estradiolinducible AvrPto-3xFLAG transgenic lines (Xiang et al. 2008), wild-type Col-0, and WS. Plants were grown on soil in growth rooms at $23^{\circ} \mathrm{C}$ during the day and $20^{\circ} \mathrm{C}$ during the night, with a 10-h light photoperiod and $75 \%$ humidity. Alternatively, seedlings were grown on vertical phytoagar plates containing one-half Murashige Skoog (MS) medium, 1.5\% sucrose, and $10 \mu \mathrm{M}$ estradiol ( $\mathrm{pH}$ 5.7) in the dark or under continuous light. Soil-grown 5-week-old plants were used for protoplast preparation, and light-grown 8-day-old seedlings on plates were used for co-IP in plants.

\section{Constructs.}

The FLS2-HA, FLS2-cYFP, AvrPto-FLAG, AvrPto-nYFP, and BSK1-HA constructs have been reported previously (Xiang et al. 2008; Zhang et al. 2010). For the FLS2-FLAG and BAK1-HA constructs, the native FLS2 and BAK1 promoter and coding region were polymerase chain reaction (PCR)-amplified and were inserted into pUC19-35S-FLAGRBS and pUC-35S-HA-RBS vector, respectively (Xiang et al. 2008). For the BAK1-FLAG construct, the $B A K 1$ cDNA was PCR-amplified and was inserted into pUC19-35S-FLAG-RBS vector. The AvrPto coding region was inserted into pUC-35SHA-RBS, resulting in an AvrPto-HA construct. For the BAK1-cYFP construct, full-length $B A K 1$ was amplified from cDNA and was cloned into the SPYCE vector (Walter et al. 2004). For HA-tagged BSK3 and CDG1 constructs, full-length $B S K 1$ and $B S K 3$ were PCR-amplified from cDNA and $C D G 1$ from genomic DNA and were cloned into 35S-HA-PUC. All constructs were verified by sequencing. Primers used for cloning are listed in Supplementary Table S1.

\section{Protoplast transfection, FRK1-LUC reporter assay, and viability assay.}

Protoplast preparation and transfection were performed as described (Asai et al. 2002), with minor modifications. Leaves of 5-week-old plants were sliced into approximately 1-mm strips and were placed in $15 \mathrm{ml}$ of enzyme solution containing $1 \%$ cellulose R10, $0.2 \%$ macerozyme R10, 0.4 M mannitol, 20 
$\mathrm{mM} \mathrm{KCl}$, and $20 \mathrm{mM}$ MES, pH 5.7, with gentle shaking (40 $\mathrm{rpm}$ ) on a rotary shaker for 2 to $3 \mathrm{~h}$. The speed was then increased to $80 \mathrm{rpm}$ for $1 \mathrm{~min}$ to release the protoplasts. The protoplasts were filtered through a mesh $(75 \mu \mathrm{m})$ and were centrifuged in a $50-\mathrm{ml}$ tube at $100 \times g$ for $2 \mathrm{~min}$, and the supernatant was carefully removed. The protoplasts were subsequently washed in 10 to $20 \mathrm{ml}$ of cold W5 solution $(154 \mathrm{mM}$ $\mathrm{NaCl}, 125 \mathrm{mM} \mathrm{CaCl}_{2}, 5 \mathrm{mM} \mathrm{KCl}$, and $2 \mathrm{mM}$ morpholineethanesulfonic acid [MES], pH5.7), were centrifuged at $100 \times g$ for $2 \mathrm{~min}$, were resuspended in $20 \mathrm{ml} \mathrm{W5}$, and were incubated on ice for $30 \mathrm{~min}$. The protoplasts were then collected by centrifugation at $100 \times g$ for 2 min and were resuspended in a solution containing $0.4 \mathrm{M}$ mannitol, $15 \mathrm{mM} \mathrm{MgCl}_{2}$, and $4 \mathrm{mM}$ MES, pH 5.7, to a final concentration of $2 \times 10^{5}$ cells $/ \mathrm{ml}$. For protoplast transfection, $10 \mu \mathrm{g}$ of plasmid DNA purified by caesium chloride ultracentrifugation was added into $200 \mu \mathrm{l}$ of protoplasts in a microfuge tube and was mixed briefly. A 220 $\mu \mathrm{4} 40 \%$ polyethylene glycol (PEG) 4,000 solution was then added and thoroughly mixed. The protoplasts were incubated at $23^{\circ} \mathrm{C}$ for $6 \mathrm{~min}$, were diluted with $0.8 \mathrm{ml}$ of $\mathrm{W} 5$ solution, and were mixed and centrifuged at $100 \times g$ for 2 min to remove PEG. The protoplasts were then resuspended gently, were diluted in $1 \mathrm{ml}$ of $\mathrm{W} 5$, and were incubated overnight under the light. For the FRK1-LUC reporter assay, protoplasts were treated with $1 \mu \mathrm{M}$ flg 22 for $3 \mathrm{~h}$ before FRK1-LUC activity was measured as described (Xiang et al. 2008). For the viability assay, a protoplast suspension $\left(10^{5}\right.$ cells $\left./ \mathrm{ml}\right)$ was mixed at a $1: 1$ ratio with $0.4 \%$ trypan blue solution, was loaded into the counting chambers of a hemocytometer, and was allowed to settle for 1 to $2 \mathrm{~min}$ before the ratio of stained to unstained cells was calculated.

\section{Co-IP assays.}

For co-IP in plants, 8-day-old light-grown seedlings were treated with $10 \mu \mathrm{M}$ estradiol on MS plates for 2 days, and total protein was isolated by homogenizing tissues with a buffer containing $50 \mathrm{mM}$ HEPES, pH 7.5, $100 \mathrm{mM} \mathrm{NaCl}, 300 \mathrm{mM}$ sucrose, $1 \mathrm{mM}$ EDTA, $5 \mathrm{mM} \mathrm{NaF}, 1 \mathrm{mM} \mathrm{Na}_{3} \mathrm{VO}_{4}$, and $1 \times$ proteinase inhibitor cocktail (Roche Molecular Systems, Inc., Mannheim, Germany). The total protein was then centrifuged at $100,000 \times g$ for $1 \mathrm{~h}$ to isolate microsomes. Membrane protein was isolated by dissolving the microsome in a buffer containing $50 \mathrm{mM}$ HEPES, pH 7.4, $150 \mathrm{mM} \mathrm{NaCl}, 0.5 \%$ Triton $\mathrm{X}-100,1 \mathrm{mM}$ EDTA, $1 \mathrm{mM} \mathrm{NaF}, 1 \mathrm{mM} \mathrm{Na} \mathrm{VO}_{4}$, and $1 \times$ proteinase inhibitor cocktail (Roche). For protoplast-based coIP, total protein was extracted from protoplasts with a buffer containing $50 \mathrm{mM}$ HEPES, $\mathrm{pH} 7.4,150 \mathrm{mM} \mathrm{NaCl}, 0.1 \%$ Triton $\mathrm{X}-100,1 \mathrm{mM}$ EDTA, $1 \mathrm{mM} \mathrm{NaF}, 1 \mathrm{mM} \mathrm{Na}_{3} \mathrm{VO}_{4}$, and $1 \times$ proteinase inhibitor cocktail (Roche). For anti-FLAG IP, approximately $2 \mathrm{mg}$ of protein was incubated with $10 \mu \mathrm{l}$ of agarose-conjugated anti-FLAG monoclonal antibody (Sigma, St. Louis) for $4 \mathrm{~h}$ at $4^{\circ} \mathrm{C}$ on a 360-degree rotary shaker. The beads were then collected and washed eight times with washing buffer (50 mM Tris- $\mathrm{HCl}, \mathrm{pH} 7.4,150 \mathrm{mM} \mathrm{NaCl}, 0.2 \%$ Triton $\mathrm{X}-100 ; 1 \mathrm{ml}$ per wash). The bound protein was eluted with 100 $\mu \mathrm{l}$ of elution buffer $(5 \mathrm{mM} 3 \times \mathrm{xLAG}$ peptide, $150 \mathrm{mM} \mathrm{NaCl}$, $50 \mathrm{mM}$ Tris- $\mathrm{HCl}, \mathrm{pH} 7.4$ ) overnight at $4^{\circ} \mathrm{C}$. For anti-HA IP, approximately $2 \mathrm{mg}$ of total protein was precleared with Protein-A-Agarose (Upstate Chemicon, Temecula, CA, U.S.A.) for $1 \mathrm{~h}$, and the protein extract was then precipitated with $2 \mu \mathrm{g}$ of anti-HA antibody and Protein-A-Agarose for $4 \mathrm{~h}$. Bound protein was eluted from beads by boiling in protein sample buffer. For anti-AvrPto IP, $\sim 2 \mathrm{mg}$ total protein was pre-cleared with Protein-A-Agarose (Upstate) for $1 \mathrm{~h}$, and the protein extract was then precipitated with $2 \mu \mathrm{g}$ anti-AvrPto antibody and Protein-A-Agarose for $4 \mathrm{hrs}$. Bound protein was eluted from beads by boiling in protein sample buffer. One third of the im- munoprecipitated protein was subjected to immunoblot analyses with anti-HA monoclonal antibody (TianGen, Beijing), anti-FLAG monoclonal antibody (Sigma), anti-FLS2 antibodies (Zhang et al. 2010), and anti-BAK1 antibody (Gao et al. 2009). Approximately $200 \mu \mathrm{g}$ of total protein was loaded as input control.

\section{BiFC assays.}

Arabidopsis protoplasts were cotransfected with cYFPtagged full-length FLS2 or BAK1 and nYFP-tagged AvrPto or empty SPYNE vector. Complementation of YFP was visualized with a confocal microscope.

\section{ACKNOWLEDGMENTS}

We thank J. Chai for critically reading the manuscript, Z. Wang for advice on experiments, P. Wu, N. Yang, and J. Ni for imaging analysis. J.-M. Zhou was supported by a grant from Chinese Ministry of Science and Technology (no. 2003-AA210080).

\section{LITERATURE CITED}

Abramovitch, R. B., Kim, Y. J., Chen, S., Dickman, M. B., and Martin, G. B. 2003. Pseudomonas type III effector AvrPtoB induces plant disease susceptibility by inhibition of host programmed cell death. EMBO (Eur. Mol. Biol. Organ.) J. 22:60-69.

Abramovitch, R. B., Janjusevic, R., Stebbins, C. E., and Martin, G. B. 2006. Type III effector AvrPtoB requires intrinsic E3 ubiquitin ligase activity to suppress plant cell death and immunity. Proc. Natl. Acad. Sci. U.S.A. 103:2851-2856.

Asai, T., Tena, G., Plotnikova, J., Willmann, M. R., Chiu, W. L., GomezGomez, L., Boller, T., Ausubel, F. M., and Sheen, J. 2002. MAP kinase signaling cascade in Arabidopsis innate immunity. Nature 415:977-983.

Block, A., Li, G., Fu, Z. Q., and Alfano, J. R. 2008. Phytopathogen type III effector weaponry and their plant targets. Curr. Opin. Plant Biol. 11:396-403.

Boller, T., and He, S. Y. 2009. Innate immunity in plants: An arms race between pattern recognition receptors in plants and effectors in microbial pathogens. Science 324:742-744.

Chinchilla, D., Bauer, Z., Regenass, M., Boller, T., and Felix, G. 2006. The Arabidopsis receptor kinase FLS2 binds flg22 and determines the specificity of flagellin perception. Plant Cell 18:465-476.

Chinchilla, D., Zipfel, C., Robatzek, S., Kemmerling, B., Nurnberger, T. Jones, J. D., Felix, G., and Boller, T. 2007. A flagellin-induced complex of the receptor FLS2 and BAK1 initiates plant defence. Nature 448:497-500

Chisholm, S. T., Coaker, G., Day, B., and Staskawicz, B. J. 2006. Host-microbe interactions: Shaping the evolution of the plant immune response. Cell 124:803-814.

Fu, Z. Q., Guo, M., Jeong, B. R., Tian, F., Elthon, T. E., Cerny, R. L., Staiger, D., and Alfano, J. R. 2007. A type III effector ADP-ribosylates RNA-binding proteins and quells plant immunity. Nature 447:284-288.

Gao, M., Wang, X., Wang, D., Xu, F., Ding, X., Zhang, Z., Bi, D., Cheng, Y. T., Chen, S., Li, X., and Zhang Y. 2009. Regulation of cell death and innate immunity by two receptor-like kinases in Arabidopsis. Cell Host Microbe 6:34-44.

Gimenez-Ibanez, S., Hann, D. R., Ntoukakis, V., Petutschnig, E., Lipka, V., and Rathjen, J. P. 2009. AvrPtoB targets the LysM receptor kinase CERK1 to promote bacterial virulence on plants. Curr. Biol. 19:423429.

Gohre, V., and Robatzek, S. 2008. Breaking the barriers: Microbial effector molecules subvert plant immunity. Annu. Rev. Phytopathol. 46:189215.

Gohre, V., Spallek, T., Haweker, H., Mersmann, S., Mentzel, T., Boller, T., de Torres, M., Mansfield, J. W., and Robatzek, S. 2008. Plant patternrecognition receptor FLS2 is directed for degradation by the bacterial ubiquitin ligase AvrPtoB. Curr. Biol. 18:1824-1832.

Heese, A., Hann, D. R., Gimenez-Ibanez, S., Jones, A. M., He, K., Li, J., Schroeder, J. I., Peck, S. C., and Rathjen, J. P. 2007. The receptor-like kinase SERK3/BAK1 is a central regulator of innate immunity in plants. Proc. Natl. Acad. Sci. U.S.A. 104:12217-12222.

Janjusevic, R., Abramovitch, R. B., Martin, G. B., and Stebbins, C. E. 2006. A bacterial inhibitor of host programmed cell death defenses is an E3 ubiquitin ligase. Science 311:222-226.

Lacombe, S., Rougon-Cardoso, A., Sherwood, E., Peeters, N., Dahlbeck, D., van Esse, H. P., Smoker, M., Rallapalli, G., Thomma, B. P., 
Staskawicz, B., Jones, J. D., and Zipfel, C. 2010. Interfamily transfer of a plant pattern-recognition receptor confers broad-spectrum bacterial resistance. Nat. Biotechnol. 28:365-369.

Larkin, M. A., Blackshields, G., Brown, N. P., Chenna, R., McGettigan, P. A., McWilliam, H., Valentin, F., Wallace, I. M., Wilm, A., Lopez R., Thompson, J. D., Gibson, T. J., and Higgins, D. G. 2007. ClustalW and ClustalX version 2. Bioinformatics 23:2947-2948.

Lu, D., Wu, S., Gao, X., Zhang, Y., Shan, L., and He, P. 2010. A receptorlike cytoplasmic kinase, BIK1, associates with a flagellin receptor complex to initiate plant innate immunity. Proc. Natl. Acad. Sci. USA. 107:496-501.

Miya, A., Albert, P., Shinya, T., Desaki, Y., Ichimura, K., Shirasu, K., Narusaka, Y., Kawakami, N., Kaku, H., and Shibuya, N. 2007. CERK1, a LysM receptor kinase, is essential for chitin elicitor signaling in Arabidopsis. Proc. Natl. Acad. Sci. U.S.A. 104:19613-19618.

Mucyn, T. S., Clemente, A., Andriotis, V. M., Balmuth, A. L., Oldroyd, G. E., Staskawicz, B. J., and Rathjen, J. P. 2006. The tomato NBARC-LRR protein Prf interacts with Pto kinase in vivo to regulate specific plant immunity. Plant Cell 18:2792-2806.

Muto, H., Yabe, N., Asami, T., Hasunuma, K., and Yamamoto, K. T. 2004. Overexpression of constitutive differential growth 1 gene, which encodes a RLCKVII-subfamily protein kinase, causes abnormal differential and elongation growth after organ differentiation in Arabidopsis. Plant Physiol. 136:3124-3133.

Noguchi, T., Fujioka, S., Choe, S., Takatsuto, S., Yoshida, S., Yuan, H., Feldmann, F. A., and Tax, F. E. 1999. Brassinosteroid-insensitive dwarf mutants of Arabidopsis accumulate brassinosteroids. Plant Physiol. 121:743-752.

Schulze, B., Mentzel, T., Jehle, A. K., Mueller, K., Beeler, S., Boller, T., Felix, G., and Chinchilla, D. 2010. Rapid heteromerization and phosphorylation of ligand-activated plant transmembrane receptors and their associated kinase BAK1. J. Biol. Chem. 285:9444-9451.

Shan, L., Thara, V. K., Martin, G. B., Zhou, J.-M., and Tang, X. 2000. The Pseudomonas AvrPto protein is differentially recognized by tomato and tobacco and is localized to the plant plasma membrane. Plant Cell 12:2323-2338.

Shan, L., He, P., Li, J., Heese, A., Peck, S. C., Nurnberger, T., Martin, G. B., and Sheen, J. 2008. Bacterial effectors target the common signaling partner BAK1 to disrupt multiple MAMP receptor-signaling complexes and impede plant immunity. Cell Host Microbe 4:17-27.

Shiu, S. H., and Bleecker, A. B. 2001. Receptor-like kinases from Arabidopsis form a monophyletic gene family related to animal receptor kinases. Proc. Natl. Acad. Sci. U.S.A. 98:10763-10768.

Tamura, K., Dudley, J., Nei, M., and Kumar, S. 2007. MEGA4: Molecular evolutionary genetics analysis (MEGA) software version 4.0. Mol. Biol. Evol. 24:1596-1599.

Tang, W., Kim, T. W., Oses-Prieto, J. A., Sun, Y., Deng, Z., Zhu, S., Wang, R., Burlingame, A. L., and Wang, Z. Y. 2008. BSKs mediate signal transduction from the receptor kinase BRI1 in Arabidopsis. Science 321:557-560.

Tang, X., Frederick, R. D., Zhou, J., Halterman, D. A., Jia, Y., and Martin, G. B. 1996. Initiation of plant disease resistance by physical interaction of AvrPto and Pto kinase. Science 274:2060-2063.

Veronese, P., Nakagami, H., Bluhm, B., Abuqamar, S., Chen, X., Salmeron, J., Dietrich, R. A., Hirt, H., and Mengiste, T. 2006. The membrane- anchored BOTRYTIS-INDUCED KINASE1 plays distinct roles in Arabidopsis resistance to necrotrophic and biotrophic pathogens. Plant Cell 18:257-273.

Walter, M., Chaban, C., Schutze, K., Batistic, O., Weckermann, K., Nake, C., Blazevic, D., Grefen, C., Schumacher, K., Oecking, C., Harter, K., and Kudla, J. 2004. Visualization of protein interactions in living plant cells using bimolecular fluorescence complementation. Plant J. 40:428438.

Wan, J., Zhang, X. C., Neece, D., Ramonell, K. M., Clough, S., Kim, S. Y., Stacey, M. G., and Stacey, G. 2008. A LysM receptor-like kinase plays a critical role in chitin signaling and fungal resistance in Arabidopsis. Plant Cell 20:471-481.

Wang, X., Kota, U., He, K., Blackburn, K., Li, J., Goshe, M. B., Huber, S. C., and Clouse, S. D. 2008. Sequential transphosphorylation of the BRI1/BAK1 receptor kinase complex impacts early events in brassinosteroid signaling. Dev. Cell 15:220-235.

Wang, Y., Li, J., Hou, S., Wang, X., Li, Y., Ren, D., Chen, S., Tang, X., and Zhou, J.-M. 2010. A Pseudomonas syringae ADP-ribosyltransferase inhibits Arabidopsis mitogen-activated protein kinase kinases. Plant Cell 22:2033-2044.

Wu, A. J., Andriotis, V. M., Durrant, M. C., and Rathjen, J. P. 2004. A patch of surface-exposed residues mediates negative regulation of immune signaling by tomato Pto kinase. Plant Cell 16:2809-2821.

Xiang, T., Zong, N., Zou, Y., Wu, Y., Zhang, J., Xing, W., Li, Y., Tang, X., Zhu, L., Chai, J., and Zhou, J.-M. 2008. Pseudomonas syringae effector AvrPto blocks innate immunity by targeting receptor kinases. Curr. Biol. 18:74-80

Xing, W., Zou, Y., Liu, Q., Liu, J., Luo, X., Huang, Q., Chen, S., Zhu, L., Bi, R., Hao, Q., Wu, J. W., Zhou J.-M., and Chai, J. 2007. The structural basis for activation of plant immunity by bacterial effector protein AvrPto. Nature 449:243-247.

Zhang, J., and Zhou, J.-M. 2010. Plant immunity triggered by microbial molecular signatures. Mol. Plant 3:783-793.

Zhang, J., Shao, F., Li, Y., Cui, H., Chen, L., Li, H., Zou, Y., Long, C., Lan, L., Chai, J., Tang, X., and Zhou, J.-M. 2007. A Pseudomonas syringae effector inactivates MAPKs to suppress PAMP-induced immunity in plants. Cell Host Microbe 1:175-185.

Zhang, J., Li, W., Xiang, T., Liu, Z., Laluk, K., Ding, X., Zou, Y., Gao, M., Zhang, X., Chen, S., Mengiste, T., Zhang, Y., and Zhou, J.-M. 2010 Receptor-like cytoplasmic kinases integrate signaling from multiple plant immune receptors and are targeted by a Pseudomonas syringae effector. Cell Host Microbe 7:290-301.

Zhou, J.-M., and Chai, J. 2008. Plant pathogenic bacterial type III effectors subdue host responses. Curr. Opin. Microbiol. 11:179-185.

Zipfel, C. 2008. Pattern-recognition receptors in plant innate immunity. Curr. Opin. Immunol. 20:10-16.

Zipfel, C. 2009. Early molecular events in PAMP-triggered immunity. Curr. Opin. Plant Biol. 12:414-420.

Zipfel, C., Robatzek, S., Navarro, L., Oakeley, E. J., Jones, J. D., Felix, G., and Boller, T. 2004. Bacterial disease resistance in Arabidopsis through flagellin perception. Nature 428:764-767.

Zipfel, C., Kunze, G., Chinchilla, D., Caniard, A., Jones, J. D., Boller, T., and Felix, G. 2006. Perception of the bacterial PAMP EF-Tu by the receptor EFR restricts Agrobacterium-mediated transformation. Cell 125:749-760. 